\title{
Toward a mature industrial practice of software test automation
}

\author{
Hong Zhu • Daniel Hoffman $\cdot$ John Hughes $\cdot$ Dianxiang Xu
}

Published online: 10 April 2014

(C) Springer Science+Business Media New York 2014

Since the launch of the first IEEE/ACM International Workshop on Automation of Software Test eight years ago, the research in the area has developed rapidly as test automation has become a mature practice widely adopted by the IT and software industry.

One of the goals that has been continuously pursued is to bridge the gap between research and practice. Many of the recent papers are closely related to the current practice in the industry reflecting the trend toward a mature community unifying both researchers and practitioners. This is also reflected in the diversity of the topics related to the practical industrial problems covered in this special section.

Authorization systems are at the heart of computer security. Antonia Bertolino et al.'s paper entitled Testing of PolPA-based Usage Control Systems is devoted to the testing of the Policy Decision Point (PDP) within the authorization system called PolPA, which enables both history-based and usage-based control of accesses. They propose two testing strategies for validating the history-based access control and the usage control functionalities, respectively. The former is based on a fault model being able to highlight the problems and vulnerabilities that could occur during the PDP implementation. The latter combines the standard technique for condition coverage with a methodology for simulating the continuous control of the PDP

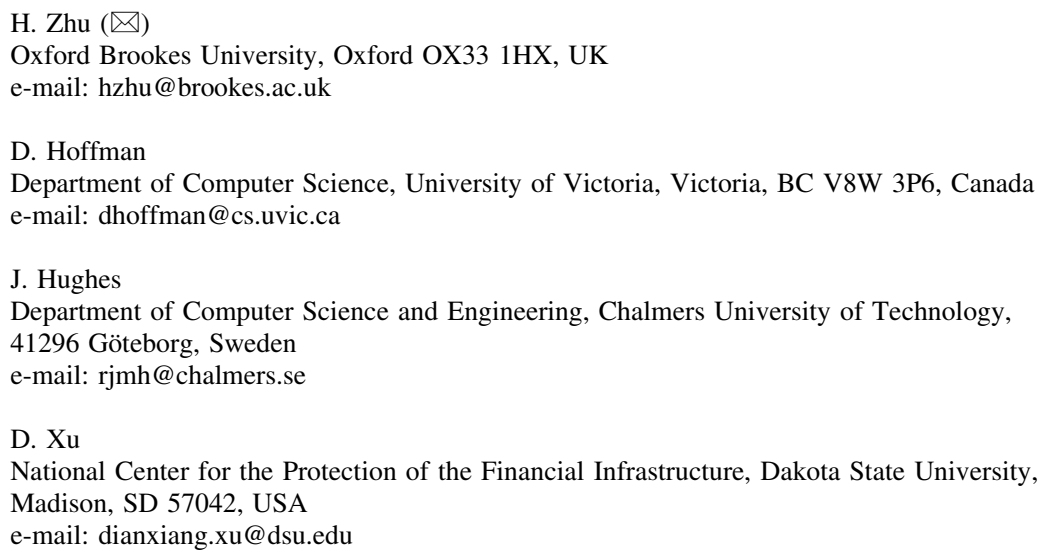


during the runtime execution. The paper reports an implementation of the strategies within a testing framework supporting the automatic generation and execution of security test suites. It also presents the results of applying the testing framework to a real case study.

Raphael Pham et al. present a video-based documentation technique of automated GUI tests to support software debugging in the paper entitled Tailoring Video Recording to Support Efficient GUI Testing and Debugging. They link the executed test instructions to the on-screen responses of the application under test. The approach captures all details relevant to debugging through a screen recording technique that is optimized for speed and memory consumption and supports browsing capabilities. The paper also reports an evaluation of the approach using an industrial experiment with 370 real-world test cases carried out in a large software company.

In the paper entitled BlackHorse: Creating Smart Test Cases from Brittle Recorded Tests, Santo Carino et al. present an approach called BlackHorse to make recorded brittle test cases more flexible and reusable by converting a recorded test case to Java code that bypasses the GUI. The paper reports the implementation of the approach in the testing environment of Research In Motion.

Test case generation is one of the key issues in software test automation. In the past years, a large number of test case generation techniques have been proposed and investigated; see (Anand et al. 2013) for a recent survey. Among the most well known are codebased test generation techniques. The paper entitled "Software Testing with Code-based Test Generators: Data and Lessons Learned from a Case Study with an Industrial Software Component" by Pietro Braione et al. studies the effectiveness of a set of state-of-theresearch test generators on a family of industrial programs. The case study demonstrated the strengths and weaknesses of the test generators used in the study. The empirical data show that the test generators can truly expose subtle (previously unknown) bugs in the subject software, and that there can be merit in using different types of test generation approaches in a complementary, even synergistic way. The authors also point out that the support for floating point arithmetic and nonlinear computations is in high demand to exploit the full potential of the prototypes based on symbolic execution.

In the paper entitled Similarity-Based Test Case Prioritization Using Ordered Sequences of Program Entities, Fang et al. address the effectiveness problem of regression testing by proposing a technique that orders program entities according to their execution frequency on test cases. Test cases are then prioritized for regression testing according to the edit distances of the ordered sequences of entities in order to maximize their diversity. The paper reports an empirical study of five open source programs. The experimental results show that their techniques can significantly increase the fault detection rate and reduce costs.

The guest co-editors are most grateful to all the reviewers. Their detailed and constructive comments greatly helped to improve the quality of the papers and are highly appreciated. The guest co-editors would also like to thank Prof. Rachel Harrison, the Editor-in-Chief of the Software Quality Journal, as well as the staff of the journal's editorial office, for their assistance in the review process of the journal special issue.

Enjoy this special section!

\section{Reference}

Anand, S., Burke, E. K., Chen T. Y., Clark, J., Cohen, M. B., Grieskamp, W., Harman, M., Harrold, M. J., McMinn, M. J. (2013) (Orchestrated and edited by A. Bertolino, J. Jenny Li, H. Zhu ). An orchestrated survey of methodologies for automated software test case generation. Journal of Systems and Software, 86(8):1978-2001. 